\title{
O ORDENAMENTO DA PESCARIA DE CAMARÕES-DE-PROFUNDIDADE (DECAPODA: ARISTEIDAE) NO SUDESTE E SUL DO BRASIL
}

\author{
DALLAGNOLO, R.*; PEREZ, J.A.A. \& PEZZUTO, P.R. \\ Universidade do Vale do Itajaí, Centro de Ciências Tecnológicas da Terra e do Mar. \\ Rua Uruguai, 458, C.P. 360, CEP 88.392-202, Itajaí - SC, Brasil. \\ *e-mail: rdallagnolo@univali.br
}

\begin{abstract}
Dallagnolo, R.; Perez, J.A.A. \& Pezzuto, P.R. 2009. Management of the deep-sea shrimp fishery (Decapoda: Aristeidae) in southern Brazil. Braz. J. Aquat. Sci. Technol. 13(2):31-43. ISSN 1808-7035. This work describes the management process established for a new deep-water $(600-800 \mathrm{~m})$ trawl fishery in Brazil, including a review of the biological elements upon which management measures were recommended. This fishery was established in slope areas of southeastern and southern sectors of Brazilian coast during the last quarter of 2002 when profitable concentrations of "carabineiro" shrimp (Aristaeopsis edwardsiana), "moruno" shrimp (Aristaeomorpha foliacea) and "alistado" shrimp (Aristeus antillensis) were found by foreign stern trawl vessels. These vessels were chartered by Brazilian companies whose contracts were supported by a development plan of the Brazilian Government. A complete fishery evaluation, including stock assessment, potential yields estimation and biomass dynamics analysis of "carabineiro" shrimp, the main species caught, was conducted and has resulted on the development of a management plan, which incorporates technical points of reference, criteria for fishery diagnosis and decision making, and exclusive management measures. The main proposed management measures include a total allowable catch of 60 ton.year ${ }^{-1}$ to be harvested by a maximum of two national vessels, operating in a fishing area rotation scheme. The plan addresses also fishing exclusion areas (that include seamounts), bathymetric and by-catch limits, fishing gear restrictions, VMS (Vessel Monitoring System), logbooks and onboard observers.
\end{abstract}

Keywords: stock assessment, fishery management, deep-sea fishery, aristeid shrimps

\section{INTRODUÇÃO}

A família Aristeidae (Ordem Decapoda, Subordem Dendrobranchiata, Superfamília Penaeoidea) é composta por camarões típicos de águas profundas, já registrados entre 100 e mais de 2.000 metros de profundidade (Sardà et al., 2004). São nove gêneros e vinte e seis espécies sendo que destas pelo menos seis apresentam importância econômica e sustentam atualmente pescarias comerciais direcionadas.

No nordeste do Atlântico Aristaeomorpha foliacea e Aristeus antennatus são os alvos de uma das mais antigas pescarias de profundidade no Mar Mediterrâneo (Sardà et al., 2003) e em Portugal as mesmas espécies e Aristaeopsis edwardsiana, fazem parte das capturas de uma pescaria multi-específica no talude continental (Figueiredo et al., 2001; Monteiro et al., 2001). Essa última espécie e Aristeus varidens são recursos explorados desde a década de 1960 na costa da Angola por embarcações espanholas (Campos-Rosado, 1974). Na parte noroeste do Atlântico, $A$. edwardsiana e Aristeus antillensis são capturados em uma pescaria sazonal no talude continental da Guiana Francesa (Guéguen, 1997, 1998, 2000, 2001). No oceano Índico, na parte noroeste da Austrália, $A$. edwardsiana e Aristeus virilis são recursos importantes de uma pescaria local (Rainer, 1992) e no Oceano Pacífico oeste, A. foliacea é capturado comercialmente nas costas sudoeste e nordeste de Taiwan (Kao et al.,1999). Todas as pescarias direcionadas a esses camarões são realizadas por arrasto-de-fundo em torno dos $700 \mathrm{~m}$ de profundidade onde, caracteristicamente, encontram-se as maiores concentrações dessas espécies.

Além de sustentarem pescarias direcionadas já estabelecidas, os camarões aristeídeos são apontados como recursos pesqueiros potenciais em várias regiões do planeta. Registros de grandes concentrações de A. edwarsiana entre Aruba, Estados Unidos e Canadá foram feitos nas décadas de 1970 e 1980, embora não se tenham registros de pescarias comerciais sobre esses estoques (Thompson et al., 1977; Markle et al., 1988). No Japão foram identificadas cinco espécies da família entre 500 e $700 \mathrm{~m}$, incluindo A. edwardsiana, A. foliacea, A. virilis, Aristeus mabahissaee Aristeus pallidicauda (Komai, 1993). No oceano Índico Suman et al. (2006) apontou $A$. edwardsiana e $A$. virilis como potenciais recursos na ilha de Sumatra, Indonésia.

No Brasil estudos taxonômicos sobre essa família, realizados em meados da década de 1990 (D'Incao, 1995, 1998) e mais recentemente na década de 2000 (Tavares \& Serejo, 2007), identificaram ao total 
nove espécies distribuídas no talude continental. Três dessas espécies tornaram-se alvos de uma pescaria direcionada em águas nacionais, o camarão-carabineiro (Aristaeopsis edwardsiana), o camarão-moruno (Aristaeomorpha foliacea) e o camarão-alistado (Aristeus antillensis), a qual tomou impulso no último trimestre de 2002 quando concentrações rentáveis foram identificadas por embarcações arrendadas de arrastode-fundo que operavam na região sudeste e sul do Brasil (Pezzuto et al., 2006a). A operação dessas embarcações foi viabilizada mediante contratos de arrendamento incentivados por meio de um plano de ocupação e exploração de águas profundas da ZEE brasileira lançado pelo Governo Federal no final da década de 1990 (Perez et al., 2003). Como forma de garantir a geração de informações que permitissem para a avaliação do potencial de sustentabilidade ecológica de pescas profundas em águas brasileiras exigiu-se de todas as embarcações arrendadas a manutenção obrigatória de sistemas de rastreamento satelital e observadores de bordo (Decreto Federal No. 2840, de 10 de novembro de 1998). Tais sistemas de monitoramento da pesca profunda permitiram o registro detalhado de todas as operações de pesca que por sua vez serviram de base para o desenvolvimento de estudos específicos visando a geração de subsídios para a elaboração de planos de ordenamento de pescarias em tais áreas (Perez et al., 2002; Pezzuto et al., 2006b; Perez et al., 2009).

A exemplo dos planos de ordenamento desenvolvidos para a pescaria do peixe-sapo (Lophius gastrophysus) (Perez et al., 2002) e dos caranguejosde-profundidade (Chaceon ramosaee Chaceon notialis) (Pezzuto et al., 2006) o presente trabalho apresenta uma síntese do processo de ordenamento da pescaria dos camarões aristeídeos no sudeste e sul do Brasil abrangendo (I) uma descrição das fontes e tipo de informações coletadas e utilizadas; (ii) a metodologia empregada para a avaliação do estoque; (iii) as bases teóricas para o ordenamento; (iv) o diagnóstico do estado atual da pescaria e $(v)$ o plano de manejo sugerido para o ano de 2008.

\section{Fontes de Informação}

Os dados utilizados na análise da pescaria dos camarões de profundidade são provenientes de duas fontes principais de informação, o Programa de Observadores de Bordo da Frota Arrendada (PROA) e o Programa de Rastreamento de Embarcações Arrendadas (PREA) (Convênio Universidade do Vale do Itajaí (GEP/UNIVALI), Ministério da Agricultura Pecuária e Abastecimento (MAPA) e Secretaria Especial de Aqüicultura e Pesca da Presidência da República (SEAP/PR).
Os dados coletados pelos observadores de bordo incluíram, a informação sobre a tecnologia de captura e processamento, e as atividades realizadas diariamente pela frota, abrangendo informações referentes a todos os lances efetuados por cada embarcação: número do lance, data e hora do lançamento e do recolhimento da rede, duração do arrasto, coordenadas geográficas e profundidades dos locais de lançamento e de recolhimento da rede, velocidade do arrasto, características físicas do petrecho utilizado e a captura em quilogramas das espécies capturadas. Os dados provenientes do PREA incluíram o detalhamento de todas as coordenadas geográficas da navegação realizada pelas embarcações pesqueiras monitoradas.

\section{Avaliação de Estoque}

Para a avaliação do estoque foram utilizados os dados obtidos entre novembro de 2002 e maio de 2007. Nesse período operaram oito embarcações arrendadas e uma embarcação nacional as quais realizaram 75 viagens de pesca objetivando a captura de camarões aristeídeos totalizando cerca de 15.000 lances de pesca e mais de 63.000 horas de arrasto.

O processo de avaliação dos estoques e a descrição da pescaria podem ser encontrados em detalhe nos trabalhos de Pezzuto et al. (2006a), Dallagnolo (2008) e Dallagnolo et al. (2009). De uma maneira resumida, esse processo incluiu: ( $($ ) a definição da área e fundos de pesca onde se concentraram as operações da frota e capturas das espécies e (ii) as estimativas de biomassa anual das espécies, feitas através da aplicação do método de área varrida (Gunderson, 1993; Sparre \& Venema, 1997) em combinação com o modelo de depleção de Leslie (Hilborn \& Walters, 1992). Nessa etapa o talude do sudeste-sul do Brasil foi dividido em três estratos latitudinais, a saber: Setor Norte, de $18^{\circ} 20^{\prime} S$ a $22^{\circ} S$, entre Espírito Santo e Rio de Janeiro; Setor Central, de 225 a $26 \div$ S, entre o Rio de janeiro e Paraná; Setor Sul, de $26^{\circ} S$ a $28^{\circ} 30^{\prime} S$. A biomassa total do sudestesul do Brasil em cada ano foi obtida através do somatório das biomassas estimadas em cada setor definido acima. Adicionalmente foi analisada a dinâmica da biomassa do camarão-carabineiro com o uso de um modelo linear generalizado (MLG) (Gavaris, 1980). Foram feitas estimativas de rendimento sustentável a partir dos parâmetros do ciclo de vida e tamanho de primeira captura das espécies (Kirkwood et al., 1994; Beddington \& Kirkwood, 2005; Perez, 2006).

\section{Bases teóricas para o Manejo das Pescarias Objetivos do Manejo e Pontos de Referência \\ O processo de manejo de uma pescaria é composto por um amplo e complexo conjunto de tarefas que, coletivamente, devem ser orientadas por objetivos}


claros e previamente definidos. De uma maneira geral os objetivos iniciais devem definir pontos conceituais de referência, concebidos no sentido de traçar os caminhos pelos quais o ordenamento deve conduzir a pescaria em questão (Cochrane, 2005).

Para o caso da pescaria direcionada aos camarões aristeídeos, o objetivo norteador das medidas de manejo foi estabelecido a partir da definição legal sobre a utilização de recursos naturais proposta no Capítulo VI "Do meio ambiente" da Constituição Brasileira de 1988 e nas diversas convenções internacionais que tratam de questões ambientais que recomendam o uso sustentável e o ordenamento do uso de recursos naturais. Dentre essas, merecem destaque a Convenção das Nações Unidas sobre os Direitos do Mar (New York, 1982), a Conferência das Nações Unidas sobre o Ambiente e o Desenvolvimento (Rio de Janeiro, 1992) e a Conferência Mundial sobre o Desenvolvimento Sustentável (Johannesburg, 2002).

Também foi inserido no processo o "Princípio da Precaução" apresentado no Artigo 7.5 do Código de Conduta para a Pesca Responsável da FAO (FAO, 1995), que imprime a necessidade de reduzir o risco de danos a um estoque pesqueiro através da adoção de medidas que levem em consideração as incertezas do sistema-pesca, e a necessidade de se implementar ações de ordenamento mesmo que o conhecimento científico seja incompleto.

Adicionalmente, levou-se em consideração o objetivo de estímulo a pesca prevista no Código de Pesca do Brasil (Decreto Lei No. 221, de 28 de fevereiro de 1967) incorporado na filosofia atual da administração pesqueira do país, que fomenta o desenvolvimento da pesca oceânica utilizando como principal instrumento 0 arrendamento de embarcações estrangeiras (IN MA No. 3,1999; IN SEAP No. 17, 2007) para transferência de tecnologia, conhecimento de mercado e distribuição de recursos valiosos, com a perspectivas de formação de uma frota oceânica nacional.

Considerando o contexto acima, se adotou o uso sustentável desses recursos e a maximização do rendimento nessa pescaria como os objetivos conceituais a serem atingidos. Para tanto, esses objetivos, essencialmente conceituais, precisam ser traduzidos em pontos técnicos de referência a serem atingidos.

Os pontos técnicos de referência, segundo Caddy \& Mahon (1996), dividem-se em Pontos de Referência Objetivos (PROs) onde se deseja chegar ou estar, e Pontos de Referência Limites (PRLs), que devem ser evitados. O ordenamento pesqueiro, portanto, deve prever e tomar ações no sentido de conduzir ou manter a pescaria/estoques nos PROs e evitar ou retirar as pescarias/estoques dos PRLs. O Rendimento Máximo Sustentável (RMS) é um ponto técnico de referência amplamente utilizado e seu uso como PRO criticado na literatura (Gulland, 1983; Hilborn \& Walters, 1992; Quinn II \& Deriso, 1999). Conceitualmente o valor de RMS deve estar relacionado com as taxas de reposição de biomassa da população, e pode ser obtido se a mortalidade por pesca for exatamente igual a mortalidade natural (M) da população em um estado de equilíbrio. Gulland (1971) o propôs através da equação:

$R M S=X \cdot M \cdot B_{\infty}$

(Equação 01)

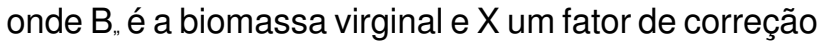
muitas vezes assumido como 0,5 . No entanto a fórmula acima acaba muitas vezes superestimando o valor de RMS pelo fato de que o pressuposto de equilíbrio (recrutamento constante e população densodependente) não é observado, além da mortalidade por pesca ser, muitas vezes, menor que M. Sua utilização mais conservativa tem sido como patamar de segurança biológica definindo o tamanho da população acima da qual seria desejável manter os níveis de biomassa do estoque explorado (Caddy \& Mahon, 1996; Pezzuto et al., 2006b).

Para o caso do camarão-carabineiro, o RMS foi calculado utilizando os parâmetros populacionais do ciclo de vida da espécie, enquanto que o nível de biomassa capaz de produzir o RMS foi determinado a partir da estimativa de biomassa virginal da espécie. Em teoria, assumindo um modelo logístico de crescimento populacional, a máxima produtividade da população, ou o rendimento máximo sustentável, se encontra no ponto médio da curva. Em termos de biomassa $\left(\mathrm{B}_{\mathrm{t}}\right)$ o modelo logístico clássico é representado pela equação:

$$
B_{t+1}=B_{t}+r \cdot B_{t} \cdot\left(1-\frac{B_{t}}{k}\right)
$$

onde $r$ representa a taxa de crescimento da população e ko parâmetro que define o valor assintótico da curva. A biomassa virginal $\left(B_{\text {.. }}\right)$ de uma população é um valor muito próximo a ko que significa que o RMS é produzido por uma biomassa $\left(B_{\mathrm{RMS}}\right)$ que se encontra exatamente na metade de $B_{n}$, conforme simplifica a equação:

$B_{R M S}=\frac{B_{\infty}}{2}$

(Equação 03)

Assumindo um estado de equilíbrio da população, a exploração ótima ideal deveria ocorrer sob um nível de biomassa exatamente igual à $B_{\mathrm{RMS}}$ removendo 0 
excedente de produção (RMS). Novamente, o equilíbrio de uma população e seus pressupostos são raramente observados e $B_{\text {RMS }}$ deve ser utilizado com cautela. Assim, definiu-se $B_{\text {RMS }}$ como um PRL, dividindo o espectro de biomassa da população em duas zonas de segurança. Além da própria biomassa absoluta do estoque estimada mediante métodos diretos (e.g. área varrida), outros indicadores propostos para o PRL utilizado foram (a) o índice de abundância produzido anualmente pelo Modelo Linear Generalizado e (b) a CPUE. No primeiro caso definiu-se como referência o coeficiente igual a 0,5 (MLG $\left.\mathrm{RMS}_{\mathrm{S}}\right)$, metade do coeficiente no ano de referência e equivalente $a B_{\text {RMS }}$. No segundo, utilizou-se a CPUE referente à biomassa da população que produz o RMS (CPUE $\left.{ }_{\text {RMS }}\right)$ estimada, experimentalmente, tomando em conta a relação:

$$
C P U E_{R M S}=q \cdot B_{R M S}
$$

onde $q$ é o coeficiente de capturabilidade. Esse coeficiente foi estimado através da relação entre a área de influência de cada unidade de esforço das redes de arrasto (1 hora de arrasto) em relação a área total de distribuição do estoque (somatório das áreas dos fundos de pesca) (ver abordagem semelhante em Pezzuto et al., 2002). De maneira precautória, em todos os PRLs foi incorporada uma "zona de alerta" prévia, correspondente a $15 \%$ dos valores de referência, de modo a: a) contemplar as incertezas nas estimativas dos PRs e b) possibilitar a tomada de decisão antes que os PRLs sejam ultrapassados. Na Tabela 1 estão sumarizados os critérios definidos acima e utilizados para o diagnóstico do estado do estoque dos camarões aristeídeos, os pontos limite de referência e as ações recomendadas.

Além do diagnóstico do estado atual da pescaria e estoque do camarão-carabineiro feitas a partir das informações levantadas no presente trabalho e utilizando os PRLs expostos acima, foram incorporadas informações biológicas dos camarões aristeídeos, conforme estudo publicado por Pezzuto \& Dias (2007), aspectos ecossistêmicos abordados nos trabalhos de Castro et al. (2006), Pires (2007) e Serejo et al. (2007) e informações sobre pescarias em áreas próximas, nos trabalhos de Perez et al. (2002; 2005), Perez \& Wahrlich (2005), Perez \& Pezzuto (2006), Pezzuto et al. (2002; 2006a; 2006b).

Tabela 1 - Zonas de identificação do estado do estoque, critérios de diagnóstico, indicadores do ponto de referência e ações recomendadas para o ordenamento da pescaria dos camarões aristeídeos. $t$, ano; $\mathrm{B}_{\mathrm{RMS}}$, Biomassa do estoque que produz o Rendimento Máximo Sustentável (RMS); $M_{\text {LG }}$, coeficiente do Modelo Linear Generalizado; $\mathrm{CPUE}_{\mathrm{RMS}}$, Captura por Unidade de Esforço na situação de biomassa que produz o RMS.

\begin{tabular}{|c|c|c|c|}
\hline Zona & Critério & $\begin{array}{l}\text { Indicador do } \\
\text { Ponto de Referência }\end{array}$ & Ação \\
\hline Zona Segura & $\begin{array}{c}\text { Biomassa } \\
\left(\mathrm{B}_{\mathrm{t}}\right) \\
\text { CPUE }_{\mathrm{t}} \\
\mathrm{GLM}_{\mathrm{t}}\end{array}$ & $\begin{array}{l}\frac{B_{t}}{B_{R M S}}>1,15 \\
\frac{C P U E_{t}}{C P U E_{R M S}}>1,15 \\
\frac{G L M_{t}}{G L M_{R M S}}>1,15\end{array}$ & $\begin{array}{l}\text { Monitoramento } \\
\text { constante }\end{array}$ \\
\hline Zona de Alerta & $\begin{array}{c}\text { Biomassa } \\
\left(\mathrm{B}_{\mathrm{t}}\right) \\
\text { CPUE }_{\mathrm{t}} \\
\mathrm{GLM}_{\mathrm{t}}\end{array}$ & $\begin{array}{c}1<\frac{B_{t}}{B_{R M S}} \leq 1,15 \\
1<\frac{C P U E_{t}}{C P U E_{R M S}} \leq 1,15 \\
1<\frac{G L M_{t}}{G L M_{R M S}} \leq 1,15\end{array}$ & $\begin{array}{c}\text { Reduzir } \\
\text { esforço/captura }\end{array}$ \\
\hline $\begin{array}{l}\text { Zona Não- } \\
\text { Segura }\end{array}$ & $\begin{array}{c}\text { Biomassa } \\
\left(\mathrm{B}_{\mathrm{t}}\right) \\
\text { CPUE }_{\mathrm{t}} \\
\mathrm{GLM}_{\mathrm{t}}\end{array}$ & $\begin{array}{c}\frac{B_{t}}{B_{R M S}} \leq 1 \\
\frac{C P U E_{t}}{C P U E_{R M S}} \leq 1 \\
\frac{G L M_{t}}{G L M_{R M S}} \leq 1\end{array}$ & $\begin{array}{c}\text { Plano de } \\
\text { Recuperação }\end{array}$ \\
\hline
\end{tabular}




\section{DIAGNÓSTICO DA PESCARIA}

Área de pesca, frota, esforço e produção

A pescaria de camarões aristeídeos iniciada durante o quarto trimestre de 2002 esteve localizada no talude da região sudeste-sul do Brasil, em profundidades variando de 500 a 1000 metros de profundidade, entre os paralelos 1820'S e 283's, abrangendo os estados do Espírito Santo a Santa Catarina (Figura 1). Nessa região, oito embarcações atuaram na pescaria, removendo um volume de aproximadamente 600 toneladas de camarões, sendo a principal espécie capturada em todos os anos o camarão-carabineiro (75\%), seguido pelo camarãomoruno (20\%) e camarão-alistado (5\%) (Pezzuto et al., 2006a; Dallagnolo, 2008; Dallagnolo et al., 2009) (Tabela 2).

No ano de 2007, houve uma significativa redução de esforço sendo que apenas duas das embarcações arrendadas e uma embarcação nacional continuaram a exploração desses recursos. As embarcações arrendadas operaram rastreadas via satélite e com a obrigação de levar um observador a bordo enquanto que a embarcação nacional operou sem nenhum desses sistemas. Entre agosto e dezembro de 2007 ambas as embarcações estrangeiras participaram do processo de renovação de arrendamento sendo autorizadas a operarem em águas brasileiras por um período de dois anos. Isso significa que ao longo do período pode-se prever a continuação do esforço de pesca de três embarcações (incluindo-se a embarcação nacional).

O histórico das operações dessas três embarcações revela que, anualmente, todas têm potencial para realizar uma média de seis viagens de pesca cada, e capturar juntas cerca de $90.000 \mathrm{~kg}$ de camarão-carabineiro, $20.000 \mathrm{~kg}$ do camarão-moruno e 1.000 kg do camarão-alistado (Tabela 3). Também são importantes as capturas médias anuais dos caranguejos-de-profundidade (Chaceon spp.), que têm superado em cada viagem os $5 \%$ da biomassa total tolerados por normativa específica para embarcações não autorizadas para esse recurso (IN SEAP-PR No 4, 2005).

\section{Estado do Estoque}

A biomassa total do camarão-carabineiro, estimada por Dallagnolo (2008) pelo método de área varrida (Sparre \& Venema, 1997), para todo o sudestesul do Brasil no ano de 2002 foi de $865.033 \mathrm{~kg} \mathrm{(+/-}$ $64.867 \mathrm{~kg}$ ) (Tabela 4). Essa biomassa foi reduzida, em menos de cinco anos de exploração a $357.216 \mathrm{~kg} \mathrm{(+/-}$ $34.643 \mathrm{~kg}$ ) em 2007. A correção das estimativas de biomassa por área varrida através dos coeficientes de abundância produzidos pelos MLGs apontou para um declínio menos acentuado embora não menos importante. Segundo essa correção, a biomassa total

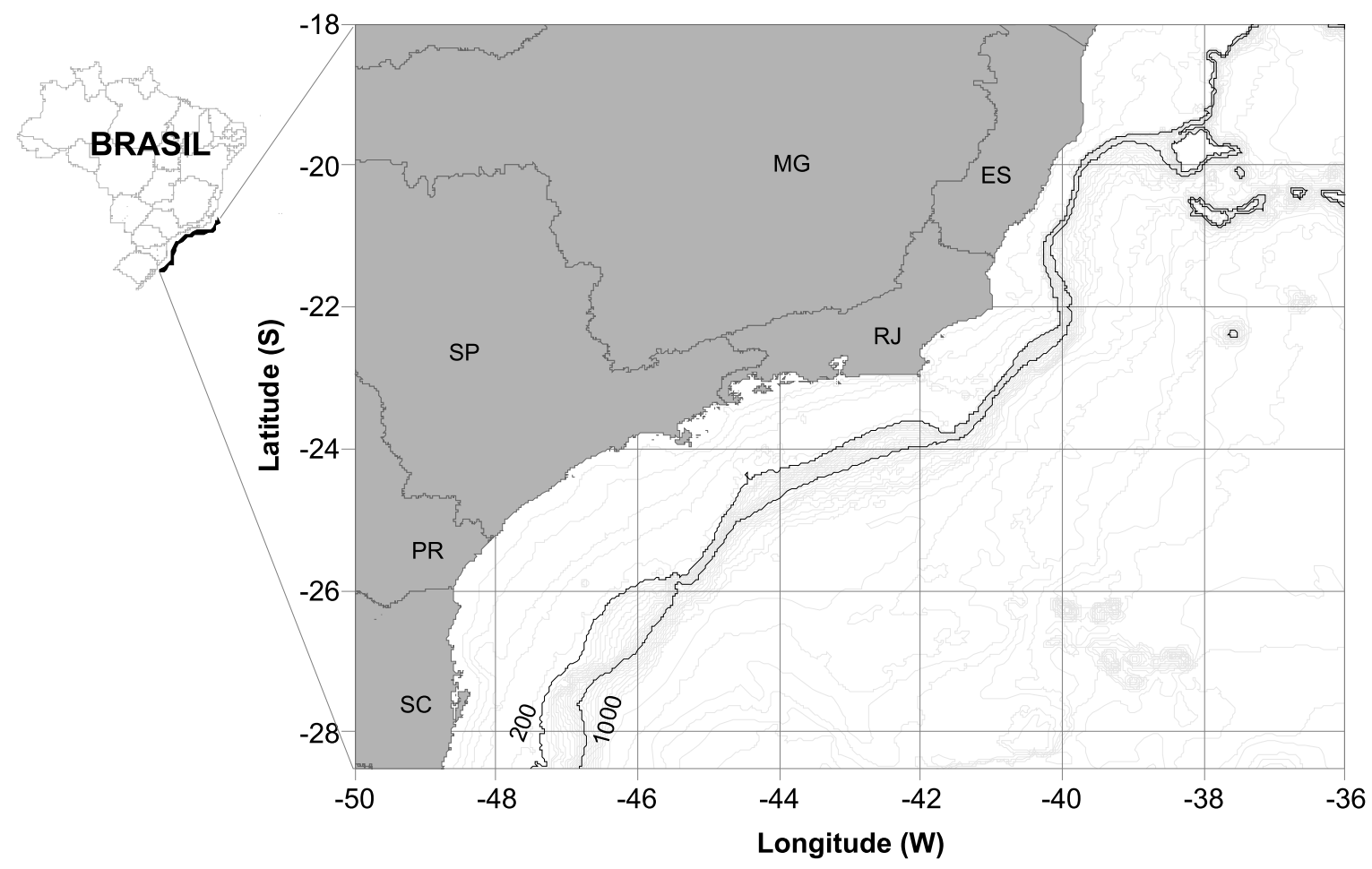

Figura 1 - Área de pesca na região sudeste-sul do Brasil, com destaque para o talude continental (linhas continuas) entre 200 m e 1000 $\mathrm{m}$ de profundidade. 
Dallagnolo et al:: O ordenamento da pescaria de camarões aristeídeos.

Tabela 2 - Sumário das capturas anuais $(\mathrm{kg})$ e esforço de pesca aplicado na pescaria de camarões aristeídeos no sudeste-sul do Brasil (Dallagnolo et al., no prelo).

\begin{tabular}{ccccccc}
\hline \hline Ano & $\begin{array}{c}\text { Aristaeopsis } \\
\text { edwardsiana }\end{array}$ & $\begin{array}{c}\text { Aristaeomorpha } \\
\text { foliacea }\end{array}$ & $\begin{array}{c}\text { Aristeus } \\
\text { antillensis }\end{array}$ & $\begin{array}{c}\text { Horas de } \\
\text { arrasto }\end{array}$ & $\begin{array}{c}\text { Número de } \\
\text { lances }\end{array}$ & $\begin{array}{c}\text { Número de } \\
\text { barcos }\end{array}$ \\
\hline 2002 & $13.021,00$ & 0,00 & 0,00 & 934,99 & 238 & 1 \\
2003 & $58.928,54$ & $4.585,38$ & 474,90 & $6.183,41$ & 1.503 & 2 \\
2004 & $81.585,11$ & $14.861,39$ & $5.489,07$ & $9.737,30$ & 2.276 & 4 \\
2005 & $182.632,91$ & $42.568,21$ & $15.828,41$ & $24.945,56$ & 6.120 & 7 \\
2006 & $99.325,30$ & $51.756,38$ & $5.365,40$ & $18.372,27$ & 4.608 & 4 \\
2007 & $19.865,00$ & $7.426,00$ & 762,00 & $3.193,78$ & 856 & 3 \\
\hline Total & $\mathbf{4 5 5 . 3 5 7 , 8 7}$ & $\mathbf{1 2 1 . 4 9 7 , 3 5}$ & $\mathbf{2 7 . 9 1 9 , 7 8}$ & $\mathbf{6 3 . 3 6 7 , 3 1}$ & $\mathbf{1 6 . 6 0 1}$ & $\mathbf{8}$ \\
\hline \hline
\end{tabular}

Tabela 3 - Capturas médias anuais das operações das embarcações de arrasto-de-fundo durante a pescaria dos camarões aristeídeos que continuaram a atividade no ano de 2007. A categoria "Peixes" inclui o peixe-sapo (Lophius gastrophysus), a merluza (Merllucius hubssi) e a abrótea-de-profundidade (Urophycis cirrata).

\begin{tabular}{lccccccc}
\hline \hline Barco & $\begin{array}{c}\text { Viagens } \\
\text { lano }\end{array}$ & $\begin{array}{c}\text { Dias de } \\
\text { pesca/ano }\end{array}$ & $\begin{array}{c}A . \\
\text { edwardsiana } \\
(\mathrm{kg})\end{array}$ & $\begin{array}{c}A . \\
\text { foliacea } \\
(\mathrm{kg})\end{array}$ & $\begin{array}{c}A . \\
\text { antillensis } \\
(\mathrm{kg})\end{array}$ & $\begin{array}{c}\text { Chaceon } \\
\text { spp. } \\
(\mathrm{kg})\end{array}$ & $\begin{array}{c}\text { Peixes } \\
(\mathrm{kg})\end{array}$ \\
\hline Mar Maria & 6 & 306 & $37.594,79$ & $7.596,87$ & 299,64 & $13.060,50$ & $4.668,00$ \\
Emb. & 6 & 279 & $20.974,00$ & $6.176,00$ & 604,00 & $2.015,00$ & $1.725,00$ \\
Nacional. & & & & & & & \\
Albamar & 6 & 301 & $30.927,17$ & $5.710,11$ & 227,44 & $7.899,75$ & $3.935,00$ \\
\hline \hline
\end{tabular}

disponível à pescaria reduziu-se para $430.169 \mathrm{~kg}(+/-$ $30.710 \mathrm{~kg}$ ) em 2007 no sudeste-sul do Brasil (Tabela 4).

A partir da estimativa da biomassa inicial, definiuse a $B_{R M S}$ do camarão-carabineiro em $432.516 \mathrm{~kg} \mathrm{(+/-}$ $32.433 \mathrm{~kg}$ ) para as áreas de pesca de todo o sudestesul do Brasil (Tabela 5). Os valores de RMS variaram entre $6,13 \%, 6,66 \%$ e $7,19 \%$ da biomassa virginal explotável o que significa valores de capturas de $53.050,1 \mathrm{~kg}, 57.640,1 \mathrm{~kg}$ e 62.230,1 kg para a espécie em todo o sudeste-sul, respectivamente. Pode-se notar que a configuração da frota atuante em 2007, tinha poder de pesca suficiente para ultrapassar com facilidade esses valores de RMS, durante o período de um ano.

Considerando o somatório das áreas dos fundos de pesca utilizados pela frota e a área de influência de cada hora de arrasto (unidade de esforço) determinouse o valor de q e a CPUE ${ }_{\text {RMS }}$ conforme a equação 04. No setor central, o de maior biomassa, a $\mathrm{CPUE}_{\mathrm{RMS}}$ foi estabelecida em 16,5 kg.hora-1, seguido por 10,1 kg.hora-1 no setor sul e 7,80 kg.hora ${ }^{-1}$ no setor norte (Tabela 5).

Tomando como base a biomassa estimada para os anos de 2006 e 2007 e os pontos limite de referência, nota-se que a biomassa do camarão-carabineiro no sudeste-sul do Brasil esteve posicionada entre 12\% (2006) e 17\% (2007) abaixo de $B_{\text {RMS }}$. A situação mais crítica foi na parcela do estoque do setor central, onde esse nível atingiu patamares de 43\% (2006) e 44\% (2007) abaixo de $B_{\text {RMS }}$ (Tabela 6). Nos demais setores a biomassa encontrou-se a $43 \%$ (2006) e $48 \%$ (2007) acima de $B_{\text {RMs }}$ no setor norte, e $59 \%$ (2006) e 30\% (2007) acima de $B_{\text {RMS }}$ no setor sul.

Utilizando como referência os coeficientes produzidos pelos MLGs, a situação do estoque no sudeste-sul aparece também em estado crítico, um pouco abaixo do $M_{L} G_{\text {RMS }}$. No setor central a situação continuou desfavorável, 30\% (2006) e 20\% (2007) abaixo de $\mathrm{MLG}_{\mathrm{RMS}}$. É importante notar que de acordo com essa referência, a biomassa no setor norte encontrou-se muito próxima a $\mathrm{MLG}_{\mathrm{RMS}}$, somente $18 \%$ acima em 2006 decaindo para apenas $8 \%$ acima no ano de 2007 . No setor sul a biomassa esteve em melhor estado, $78 \%$ acima de $M_{L G}$ em 2006 e 62\% acima em 2007 (Tabela 6). A Figura 2 apresenta graficamente as estimativas de biomassa por área varrida e a correção destas estimativas pelos coeficientes dos MLGs para toda a região Sudeste-Sul, ressaltando o nível crítico atual do estoque do camarão-carabineiro no sudestesul do Brasil em relação a $B_{\text {RMS }}$. 
Tabela 4 - Estimativas de biomassa $(\mathrm{kg})$ do camarão-carabineiro (Aristaeopsis edwardsiana) a partir do método de área varrida para as parcelas do estoque distribuídas nos três setores de operação e para todo o Sudeste-Sul do Brasil. Essas estimativas corrigidas pelos coeficientes produzidos pelos Modelos Lineares Generalizados (MLG) s de cada setor são apresentadas no lado direito da tabela. Entre parênteses estão os valores de intervalo de confiança (95\%).

\begin{tabular}{ccccc|cccc}
\hline \hline \multicolumn{5}{c|}{ Área Varrida } & \multicolumn{4}{c}{ Área varrida x Coeficientes MLG } \\
\hline \multirow{2}{*}{ Ano } & Biomassa & Biomassa & Biomassa & Biomassa & Biomassa & Biomassa & Biomassa & Biomassa \\
& Central & Norte & Sul & Total & Central & Norte & Sul & Total \\
\hline \multirow{4}{*}{2002} & $580.650,3$ & $120.150,2$ & $164.232,8$ & $865.033,3$ & $580.650,3$ & $120.150,2$ & $164.232,8$ & $865.033,3$ \\
& $(+/-$ & $(+/-$ & $(+/-$ & $(+/-$ & $(+/-$ & $(+/-$ & $(+/-$ & $(+/-64.867,9)$ \\
& $48.174,9)$ & $7.707,7)$ & $8.985,3)$ & $64.867,9)$ & $48.174,9)$ & $7.707,7)$ & $8.985,3)$ & \\
& $420.183,0$ & $120.150,2$ & $164.232,8$ & $704.566,0$ & $354.196,7$ & $120.150,2$ & $164.232,8$ & $638.579,7$ \\
2003 & $(+/-$ & $(+/-$ & $(+/-$ & $(+/-$ & $(+/-$ & $(+/-$ & $(+/-$ & $(+/-46.079,7)$ \\
& $32.133,3)$ & $7.707,7)$ & $8.985,3)$ & $48.826,3)$ & $29.386,7)$ & $7.707,7)$ & $8.985,3)$ & \\
& $276.158,1$ & $120.150,2$ & $164.232,8$ & $560.541,1$ & $209.034,1$ & $120.150,2$ & $164.232,8$ & $493.417,1$ \\
2004 & $(+/-$ & $(+/-$ & $(+/-$ & $(+/-$ & $(+/-$ & $(+/-$ & $(+/-$ & $(+/-34.036,0)$ \\
& $17.272,2)$ & $7.707,7)$ & $8.985,3)$ & $33.965,2)$ & $17.343,7)$ & $7.707,7)$ & $8.985,3)$ & \\
& $239.292,3$ & $90.562,57$ & $164.232,8$ & $494.087,7$ & $301.938,2$ & $78.097,61$ & $164.232,8$ & $544.268,6$ \\
2005 & $(+/-$ & $(+/-$ & $(+/-$ & $(+/-$ & $(+/-$ & $(+/-$ & $(+/-$ & $(+/-39.046,3)$ \\
& $9.716,1)$ & $4.859,6)$ & $8.985,3)$ & $23.561,0)$ & $25.050,9)$ & $5.010,0)$ & $8.985,3)$ & \\
& $165.545,4$ & $85.693,49$ & $130.522,6$ & $381.761,5$ & $203.227,6$ & $70.888,6$ & $146.167,2$ & $420.283,4$ \\
2006 & $(+/-$ & $(+/-$ & $(+/-$ & $(+/-$ & $(+/-$ & $(+/-$ & $(+/-$ & $(+/-29.405,7)$ \\
& $7.904,1)$ & $7.514,0)$ & $7.655,8)$ & $23.073,9)$ & $16.861,2)$ & $4.547,6)$ & $7.996,9)$ & \\
& $161.898,0$ & $88.665,82$ & $106.653,1$ & $357.216,9$ & $232.260,1$ & $64.881,09$ & $133.028,6$ & $430.169,8$ \\
2007 & $(+/-$ & $(+/-$ & $(+/-$ & $(+/-$ & $(+/-$ & $(+/-$ & $(+/-$ & $(+/-30.710,2)$ \\
& $16.024,0)$ & $10.602,5)$ & $8.016,9)$ & $34.643,4)$ & $19.270,0)$ & $4.162,2)$ & $7.278,1)$ & $(+2)$ \\
\hline \hline
\end{tabular}

Tabela 5 - Pontos Referência Limites calculados para o diagnóstico do estado do estoque do camarão-carabineiro (Aristaeopsis edwardsiana). Apresentam-se as estimativas para as parcela do estoque em cada setor de operação da frota (Central, Norte e Sul) e para todo o sudeste-sul do Brasil. q. coeficiente de capturabilidade; Ver tabela 1 para definição das demais variáveis.

\begin{tabular}{lcccccc}
\hline Setor & $\begin{array}{c}\text { Área dos } \\
\text { Fundos } \\
\left(\mathrm{km}^{2}\right)\end{array}$ & $\begin{array}{c}\text { Área de atuação } \\
\text { da rede } \\
\left(\mathrm{km}^{2}\right)\end{array}$ & $q$ & $\begin{array}{c}\mathrm{B}_{\mathrm{RMS}} \\
(\mathrm{kg})\end{array}$ & MLG $_{\mathrm{RMS}}$ & $\begin{array}{c}\mathrm{CPUE}_{\mathrm{RMS}} \\
\left.\left(\mathrm{kg}_{\mathrm{hora}}\right)^{-1}\right)\end{array}$ \\
\hline Central & $2.792,11$ & 0,1588 & 0,0000569 & $290.325,2$ & 0,5 & 16,51 \\
Norte & $1.180,00$ & 0,1533 & 0,0001300 & $60.075,1$ & 0,5 & 7,80 \\
Sul & $1.354,98$ & 0,1667 & 0,0001230 & $82.116,4$ & 0,5 & 10,10 \\
Sudeste-Sul & $5.327,09$ & 0,1574 & 0,0000295 & $432.516,6$ & 0,5 & 12,78 \\
\hline \hline
\end{tabular}

De acordo com as CPUEs estimadas, a situação da biomassa no sudeste-sul era mais crítica do que aquela apontada pelas outras referências. Para todo o sudeste-sul a CPUE obtida no ano de 2006 esteve 58\% abaixo da CPUE ${ }_{\text {RMS }}$ e $51 \%$ abaixo em 2007. Novamente o setor central possuia a parcela do estoque em pior estado, com uma CPUE situada $72 \%$ (2006) e 67\% (2007) abaixo da CPUE ${ }_{\text {RMS }}$. Nos setores sul e norte, as CPUEs estavam muito abaixo de CPUE $_{\text {RMs }}$. No setor sul, o nível das CPUEs obtidas foi $38 \%$ abaixo da CPUE $_{\text {RMS }}$ em 2006 e 2007, e no setor, norte $21 \%$ em

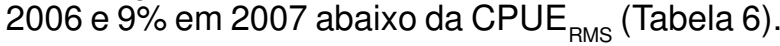

Ficou claro que o estoque de camarão-carabineiro nas áreas de pesca do talude do sudeste-sul do Brasil encontra-se num estado geral de insegurança biológica, independentemente do indicador do ponto técnico de referência considerado. Esse diagnóstico se agrava ainda mais se a parcela distribuída no setor central é considerada separadamente. O referido quadro aponta, portanto, para a tomada de ações que visem à recuperação do estoque.

A própria manutenção do esforço de pesca atual parece ser incompatível com a sustentabilidade do estoque devido ao elevado poder de pesca das três embarcações atualmente engajadas na pescaria e as altas taxas de remoção que são capazes de aplicar juntas (Tabela 3).

\section{Aspectos biológicos dos recursos-alvo}

Observou-se nos rendimentos trimestrais, uma variação sazonal bem marcada nas abundâncias do camarão-carabineiro, com picos de abundância ocorrendo durante o quarto trimestre do ano (Dallagnolo et al., 2009). Essa variação pode estar associada com o aumento da atividade reprodutiva da espécie no mesmo período (Pezzuto \& Dias, 2007). Isso tem 
Dallagnolo et al:: O ordenamento da pescaria de camarões aristeídeos.

Tabela 6 - Indicadores do estado do estoque do camarão-carabineiro (Aristaeopsis edwardsiana) em cada setor de operação da frota (Central, Norte, Sul) e para todo o sudeste-sul do Brasil em 2006 e 2007. t, ano. Ver tabela 1 para definição das demais variáveis.

\begin{tabular}{cccccccc}
\hline Setor & Ano & $\begin{array}{c}\mathrm{B}_{\mathrm{t}} \\
(\mathrm{kg})\end{array}$ & $\mathrm{MLG}_{\mathrm{t}}$ & $\begin{array}{c}\mathrm{CPUE} \\
\left(\mathrm{kg} \cdot \mathrm{hora}^{-1}\right)\end{array}$ & $\mathrm{B}_{\mathrm{t}} \mathrm{B}_{\mathrm{RMS}}$ & $\begin{array}{c}\mathrm{MLG}_{\mathrm{t}} \\
\mathrm{MLG}_{\mathrm{RMS}}\end{array}$ & $\begin{array}{c}\mathrm{CPUE}_{\mathrm{t}} \\
/ \mathrm{CPPUE}_{\mathrm{RMS}}\end{array}$ \\
\hline \multirow{2}{*}{ Central } & 2006 & $165.545,4$ & 0,35 & 4,70 & 0,57 & 0,70 & 0,28 \\
& 2007 & $161.898,0$ & 0,40 & 5,41 & 0,56 & 0,80 & 0,33 \\
\multirow{3}{*}{ Norte } & 2006 & $85.693,5$ & 0,59 & 6,14 & 1,43 & 1,18 & 0,79 \\
& 2007 & $88.665,8$ & 0,54 & 7,08 & 1,48 & 1,08 & 0,91 \\
\multirow{2}{*}{ Sul } & 2006 & $130.522,6$ & 0,89 & 6,25 & 1,59 & 1,78 & 0,62 \\
\multirow{2}{*}{$\begin{array}{c}\text { Sudeste- } \\
\text { Sul }\end{array}$} & 2007 & $106.653,1$ & 0,81 & 6,31 & 1,30 & 1,62 & 0,62 \\
\hline \hline
\end{tabular}

implicações importantes, pois o aumento de abundância do quarto trimestre é um fato conhecido das embarcações, o que pode resultar em um aumento de esforço nesse período. Outro aspecto biológico relevante são os tamanhos de primeira captura atuais (Dallagnolo, 2008), que para as três espécies superam em alguns centímetros o tamanho de primeira maturação sexual calculado por Pezzuto \& Dias (2007).

No que se refere aos aspectos acima, a pescaria não demonstra comportamento crítico, porém ressaltam-se os potenciais benefícios em se limitar a concentração do esforço durante o quarto trimestre do ano e se estabelecer o tamanho atual das malhas no ensacador da rede $(60 \mathrm{~mm})$ como o tamanho mínimo permitido, de forma a assegurar a reprodução das espécies.

\section{Aspectos ecológicos}

Considerando os dois setores onde o camarãomoruno aparece nas capturas (central e norte), a biomassa estimada no ano de 2002, de cerca de $99.831,5 \mathrm{~kg}(+/-35.272 \mathrm{~kg})$ passou para cerca de $251.336,5 \mathrm{~kg}$ (+/- $251.336 \mathrm{~kg})$ em 2007. O aumento expressivo da biomassa do camarão-moruno nos mesmos fundos onde ocorreram as quedas contínuas da biomassa do camarão-carabineiro pode ser uma evidência de competição entre essas espécies. Tal fato pode ser favorecido tanto pelas características reprodutivas do camarão-moruno, que, segundo Pezzuto \& Dias (2007), está apto a reproduzir ao longo de todo o ano, como pelo alívio da competição devido as remoções de camarão-carabineiro feitas pela frota (Dallagnolo et al., 2009).

Nos fundos de pesca utilizados pela frota existe uma grande diversidade de populações vulneráveis à pesca de arrasto profundo que, em sua grande maioria, são de baixa resiliência (Serejo et al., 2007). Existem evidências de que alguns trechos do talude, na faixa de ocorrência dos camarões aristeídeos, não foram arrastados, ou foram pouco arrastados pelas embarcações devido à presença de recifes de corais de profundidade. De fato, Castro et al. (2006) demonstraram a presença dessas formações ao longo do talude da região sudeste-sul do Brasil. Em uma revisão recente, Pires (2007) sumarizou cerca de 56 espécies de corais de profundidade no estrato batimétrico utilizado pela frota na captura desses camarões, incluindo o Banco Besnard, primeiro e maior monte submarino da Cadeia Vitória-Trindade, em frente ao estado do Espírito Santo. Nesse banco está localizado o fundo de pesca N3 e no trabalho de Pires (2007), uma das novas espécies de corais azooxantelados descritas, Flabellum cf. alabastrum, foi removida pela embarcação Kayar durante operações em $686 \mathrm{~m}$ de profundidade no ano de 2004. De maneira extensa na literatura são apontadas as fragilidades de montes submarinos e espécies que os habitam (Koslow, 1997; Koslow et al., 2001; Roberts, 2002) e atualmente, existem fortes recomendações para a incorporação dessas feições em áreas de exclusão da pesca (FAO, 2007). Perez (2007) em um trabalho que apresentou o processo de definição de Áreas de Exclusão da Pesca profunda no Brasil discutiu a necessidade de proteção de montes submarinos da região nordeste utilizados para uma pescaria de arrasto de fundo sobre o chernenegro (Epinephelus nigritus). Segundo o autor, em cerca de um mês de exploração uma única embarcação removeu entre 65 e $80 \%$ da biomassa da espécie no topo desses montes.

Também é importante citar a ocorrência de outros recursos pesqueiros de alto valor econômico nos fundos de arrasto de camarões aristeídeos, conforme apontaram Pezzuto et al. (2006a) em seu estudo sobre a fase inicial dessa pescaria. Segundo esses autores, cerca de $22 \%$ da captura desembarcada pelos arrasteiros arrendados era composta pelo caranguejoreal, Chaceon ramosae, alvo da pescaria arrendada de covos entre $27^{\circ}$ e $30^{\circ} \mathrm{S}$. Além disso, distribui-se ao sul 

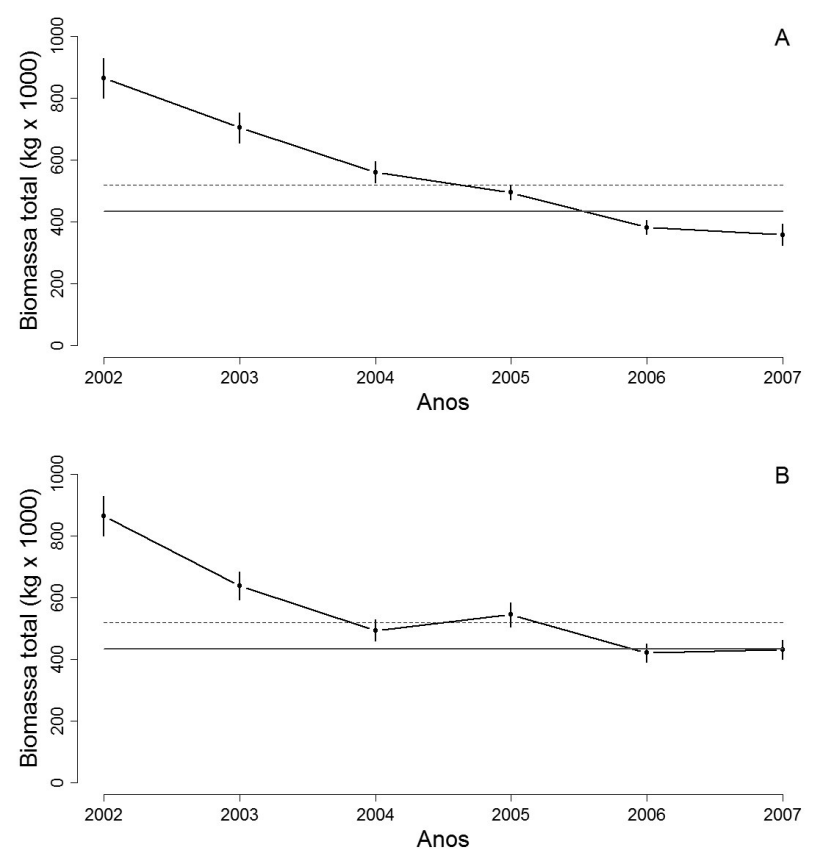

Figura 2 - Variação anual e intervalo de confiança da biomassa total do camarão-carabineiro (Aristaeopsis edwardsiana) no sudeste-sul do Brasil, estimada a partir do método de área varrida (A) e padronizada pelos coeficientes dos modelos lineares generalizados ajustados a cada setor (B). A linha horizontal contínua define o Ponto Referência Limite $\left(B_{\mathrm{RMS}}\right)$. A linha horizontal traceja define a Zona de Alerta.

de $33^{\circ} \mathrm{S}$ parte do estoque do caranguejo-vermelho Chaceon notialis, explorado em águas brasileiras por embarcações de covos e em águas uruguaias (ao sul de 34ํㅇ's) por embarcações uruguaias de covos. Nessa última região ainda não se observaram explorações comerciais de camarões aristeídeos pelas embarcações estudadas nesse trabalho, no entanto, operações destas podem potencialmente intensificar as taxas de mortalidade por pesca atualmente aplicadas nesse estoque. Da mesma forma, capturas no talude médio de peixes de valor como o peixe-sapo, a merluza e a abrótea-de-profundidade foram observadas no desenvolvimento da pesca de arrasto profunda no Brasil. Atualmente tais recursos são alvos de uma numerosa frota nacional de arrasto e emalhe-de-fundo (Perez \& Pezzuto, 2006).

Assim, de acordo com os aspectos ecológicos apresentados, pode-se generalizar que: ( $I$ ) a pescaria em discussão pode causar, ou já causou, um desequilíbrio na dominância dos recursos-alvo nos fundos de pesca; (ii) a fauna acompanhante dessa pescaria é composta tanto por organismos valiosos para outras pescarias como por corais e outros organismos frágeis, sem valor comercial mas de grande valor ecológico; e (iii) existem operações comerciais regulares em montes submarinos, onde os dois itens anteriores tornam-se recorrentes. Assim, conclui-se que essa atividade causa danos ambientais em diferentes escalas ainda pouco dimensionadas, mas que vão além da sobreexplotação do recurso-alvo.

\section{ELEMENTOS PARA O PLANO DE MANEJO}

Com base nas informações apresentadas acima uma proposta de plano de manejo para a pescaria de camarões-de-profundidade foi elaborada contendo (I) os elementos conceituais, (ii) as recomendações (pontos de referência, estratégias e táticas de manejo) propostas e os (iii) mecanismos de reavaliação do plano.

Os elementos conceituais, previamente discutidos, consideraram a sustentabilidade biológica dos recursos-alvo, a maximização dos rendimentos econômicos, a minimização de impactos colaterais, e os princípios da precaução e do manejo ecossistêmico (NRC, 1999). As recomendações são apresentadas resumidamente na Tabela 7 , onde constam os elementos do manejo gerados a partir dos elementos conceituais e do diagnóstico da pescaria.

Foram incluídos elementos básicos de manejo como: permissionamento específico, delimitação da área de pesca e unidade de manejo, definição da temporada de pesca, da modalidade permitida e das espécies-alvos e acessórias (Recomendações 1 a 7 ). Incluíram-se cotas de capturas anuais e trimestrais (Recomendação 8) em concordância com as disposições de norma específica (IN SEAP/PR No. 15, 2008) que garante o controle dos referidos limites e do esforço de pesca, indiretamente atrelado a limitação do número de embarcações nessa pescaria (Recomendação 9). Restrições tecnológicas foram inseridas no âmbito da forma de entralhamento das redes (Recomendação 10) e no tamanho mínimo das malhas utilizadas no ensacador (Recomendação 11). Atenção especial foi dada à minimização de impactos colaterais e ao princípio do manejo ecossistêmico, quando da criação de áreas de exclusão, proibição de pesca em montes submarinos e fundos coralíneos, e limitação da captura da fauna acompanhante (Recomendações 12 a 16). Por fim, medidas de controle de todas essas recomendações foram criadas com base na obrigatoriedade da entrega de Mapas de Bordo e, principalmente, pela presença de Observadores de Bordo e de Rastreamento Satelital em 100\% das viagens de pesca (Recomendação 17).

A última medida proposta foi a criação de um mecanismo de reavaliação do estado da pescaria de cada espécie-alvo, permitindo a readequação do plano em caso de necessidade. Para tal adotou-se a biomassa do estoque que produz o Rendimento Máximo Sustentável $\left(\mathrm{B}_{\mathrm{RMS}}\right)$ como Ponto de Referência Limite 
Tabela 7 - Elementos do manejo a recomendações elaboradas para o ordenamento da pescaria dos camarões aristeídeos na costa sudeste-sul do Brasil.

Elementos do manejo

1. Permissionamento

2. Área de pesca - limites latitudinais

3. Área de pesca - limites batimétricos

5. Temporada de Pesca

6. Modalidade de pesca

7. Espécies-alvo e acessórias

8. Cota de captura

9. Tamanho da Frota

10. Restrição de petrecho

11. Tamanho de malha

12. Limite de fauna acompanhante

13. Qualificação de capturas incidentais

14. Gestão de resíduos

15. Áreas de exclusão

16. Rotação de áreas

17. Medidas de controle

${ }^{a}$ Área de exclusão é definida pelas coordenadas $23^{\circ} 40^{\prime} \mathrm{S} / 44^{\circ} 00^{\prime} \mathrm{W}$

Aristaeopsis edwardsiana foliacea; Aristeus antillensis.

Cota anual de 60 t.ano $^{-1}$ considerando as três espécies; Cota individual e não transferí vel de 7,5 t.trimestre ${ }^{-1}$ para cada barco.

Duas embarcações nacionais.

Deve operar sem sobre -saco no ensacador e com o conjunto completo de portas, tralhas, bóias, redes e ensacadores fechados.

Malhas no ensacador com tamanho mínimo de $60 \mathrm{~mm}$ medidos entre nós opostos com a malha esticada.

Chaceon spp. em 15\%; Lophius gastrophysus em $5 \%$. Ambos percentuais em relação a captura total das espécies-alvo e acessórias.

Tornar-se obrigatório o reporte de captura de corais pétreos, córneos e gorgônias de profundidade, incluindo sua posição geográfica.

Armazenamento dos resíduos sólidos não biodegradáveis a bordo para posterior destinação adequada em terra

$\mathrm{SE}^{\mathrm{a}}$; montes submarinos; fundos coralíneos; áreas ao norte de $21^{\circ} \mathrm{S}^{\mathrm{b}}$.

Ao norte e ao sul da área de exclusão ficando proibida a operação das duas embarcações na mesma área ao mesmo tempo.

Observadores de bordo em $100 \%$ das viagens;

Rastreamento por satélite em $100 \%$ das viagens; Mapas de Bordo de cada viagem.

$24^{\circ} 15^{\prime} \mathrm{S} / 43^{\circ} 30^{\prime} \mathrm{W}-25^{\circ} 00^{\prime} \mathrm{S} / 44^{\circ} 30^{\prime} \mathrm{W}$

${ }^{b}$ Área deve ser excluída da pesca no momento em que a captura de $A$. antillensis atingir 4,4 toneladas. 
(PRL) expresso pelos três indicadores previamente apresentados. As ações de manejo a serem aplicadas a pesca dos camarões aristeídeos deveriam seguir as regras de decisão listadas na Tabela 1. Na existência de dois ou mais indicadores de abundância do estoque, deveriam ser adotados para efeito de decisão aqueles que oferecerem o cenário mais conservativo para o estoque.

Essa proposta foi discutida no âmbito do Subcomitê Científico (SCC) do Comitê Permanente de Gestão dos Recursos Demersais (CPG/ Demersais), órgão consultivo da Secretaria Especial de Aqüicultura e Pesca da Presidência da República (SEAP/PR). Posteriormente foi apresentado e discutido por cientistas, representantes do governo e empresas de pesca no plenário da $7^{\text {a }}$ Sessão Ordinária do CPG/ Demersais realizada em Brasília, Distrito Federal, nos dias 25 e 26 de agosto de 2008 permanecendo em discussão ao menos até junho de 2009. Apesar dos esforços descritos neste trabalho, até esse momento, a pescaria tem prosseguido de forma descontrolada.

\section{AGRADECIMENTOS}

Os autores agradecem a todos observadores de bordo da frota arrendada pela dedicação na coleta dos dados utilizados nesse trabalho. Igualmente importante foi a colaboração dos administradores, comandantes e tripulações das embarcações de pesca. Também, a toda a equipe do Grupo de Estudos Pesqueiros da Universidade do Vale do Itajaí, no processamento e armazenamento dessas informações e ao Governo Federal pelo suporte financeiro para a execução deste trabalho através dos convênios estabelecidos (MA/ SARC/03/2000; MAPA/SARC/DPA/003/2001; MAPA/ SARC/DENACOOP/ 176/2002; SEAP/PR/001/2003; SEAP/PR/078/2004; SEAP/PR/064/2005; SEAP/PR/ 027/2007). J.A.A.P. e P.R.P. receberam apoio do CNPq através de bolsas de produtividade (Processos: 306184/ 2007-9 e 310820/2006-5 respectivamente).

\section{REFERÊNCIAS}

Beddington, J.R. \& Kirkwood, G.P. 2005. The estimation of potential yield and stock status using life-history parameters. Philosophical Transactions of the Royal Society, Biological Sciences. 360:163-170.

Caddy, J. F. \& Mahon, R. 1996. Puntos de referencia para la ordenación pesquera. Roma: FAO Documento Técnico de Pesca, 347. 109p.

Campos-Rosado, J. M. 1974. Catches of deep sea shrimps by Spanish trawlers of Angola, 1967-1972. Collection of Scientific Papers ICSEAF. 1:78-101.
Castro, B. M.; Lorenzzetti, J. A.; Silveira, I. C. A. \& Miranda, L. B. 2006. Estrutura termohalina e circulação na região entre o Cabo de São Tomé (RJ) e o Chuí (RS). In: Rossi-Wongtschowsky, C. L. B. \& Madureira, L. S. P. (Eds.). O ambiente oceanográfico da plataforma continental e do talude da região sudeste-sul do Brasil. São Paulo: Editora da Universidade de São Paulo. 11-120pp.

Cochrane, K. L. 2005. Guía del administrador pesquero. Roma: FAO Documento Técnico de Pesca, 424. $231 p$.

Dallagnolo, R. 2008. A pesca de camarões-deprofundidade (Aristeidae) na região sudeste-sul do Brasil: avaliação da dinâmica de biomassa como ferramenta para gestão. Tese de Mestrado. Universidade do Vale do Itajaí. 215p.

Dallagnolo, R.; Perez, J. A. A.; Pezzuto, P. R. \& Wahrlich, R. 2009 (no prelo). The deep-sea shrimp fishery off Brazil (Decapoda: Aristeidae): development and presents status. Latin American Journal of Aquatic Research, 37(2):000-000.

D'Incao, F. 1995. Taxonomia, padrões distribucionais e ecológico dos Dendrobranchiata (Crustacea: Decapoda) do Brasil e Atlântico Ocidental. Tese de Doutorado. Universidade Federal do Paraná. 365p.

D'Incao, F. 1998. The Brazilian species of the family Aristeidae Wood-Mason (Crustacea: Decapoda). Journal of Natural History. 32:1509-1518.

FAO, 1995. Código de conducta para la pesca reponsable. Roma: FAO Documento Técnico de Pesca, 64p.

FAO, 2007. Draft International Guidelines on the Management of Deep-sea Fisheries in the High Seas. Roma: FAO Fisheries Technical Paper, 32p.

Figueiredo, M. J.; Figueiredo, I. \& Machado, P.B. 2001. Deep-water shrimps (Crustacea: Decapoda) from off the Portuguese continental slope: an alternative future resource? Fisheries Research. 51:321-326.

Gavaris, S. 1980. Use of a multiplicative model to estimate catch rate and effort form commercial data. Can. J. Fish. Aquat. Sci., vol. 37:2272-2275.

Guéguen, F. 1997. Étude biométrique de deux espèces de crevettes profondes exploitées en Guyane Française. Biologie et Pathologie Animales. 320:899-900.

Guéguen, F. 1998. Biologie de la crevette profonde Plesiopenaeus edwardsianus en Guyane Française. Biologie Animale. 321:757-770.

Guéguen, F. 2000. Distribution et abondance des crustacés decapods du talus continental (200-900 m) de Guyane Française. Crustaceana. 73(6):685703.

Guéguen, F. 2001. Notes sur la biologie de la crevette de profondeur Aristeus antillensis en Guyane Française. Life Sciences. 324:689-700. 
Gulland, J. A. 1971. The fish resources of the ocean. Fishing News (Books) Limited Publishers, West Byfleet, Surrey. 255p.

Gulland, J. A. 1983. Fish stock assessment: a manual of basic methods. New York: John Wiley \& Sons. $223 p$.

Gunderson, D. R. 1993. Surveys of fisheries resources. New York: John Wiley \& Sons. 248p.

Hilborn, R. \& Walters, C.J. 1992. Quantitative fisheries stock assessment: choice, dynamics and uncertainty. New York: Chapman \& Hall. 570p.

Kao, H. C.; Chan, T. Y. \& Yu, H. P. 1999. Ovary development of the deep-water shrimp Aristaeomorpha foliacea (Risso, 1826) (Crustacea: Decapoda: Aristeidae) from Taiwan. Zoological Studies. 38:373-378.

Komai, T. 1993. Deep-sea shrimps of the family Aristeidae (Decapoda: Dendrobranchiata) from northern Japan, with the description of a new species of the genus Aristeus. Crustacean Research. 22:2134.

Koslow, J. A. 1997. Seamounts and the ecology of deepsea fisheries. American Scientist. 85:168-176.

Koslow, J. A.; Gowlett-Holmes, K.; Lowry, J.K.; O’hara, T.; Poore, G.C.B. \& Williams, A. 2001. Seamount benthic macrofauna off southern Tasmania: community structure and impacts of trawling. Marine Ecology Progress Series. 213:111-125.

Kirkwood, G.P.; Beddington, J.R. \& Rosouw, J.A. 1994. Harvesting species of different lifespans. In: Edwards, P.J.; May, R. \& Webb, N.R. (Eds.) Largescale ecology and conservation biology. Oxford: Blackwell Scientific Publications, 199-227pp.

Markle, D.; Dadswell, M. J. \& Halliday, R. G. 1988. Demersal fish and decapod crustacean fauna of the upper continental slope of Nova Scotia from LaHave to St Pierre Banks. Canadian Journal of Zoology. 66(9):1952-1960.

Monteiro, P.; Araújo, A.; Erzini, K. \& Castro, M. 2001. Discards of the Algarve (southern Portugal) crustacean trawl fishery. Hydrobiologia. 449:267277.

NRC, 1999. Sustaining marine fisheries. Washington: National Academy Press. 164p.

Perez, J.A.A. 2006. Potenciais de rendimento dos alvos da pesca de arrasto de talude do sudeste e sul do Brasil estimados a partir dos parâmetros do ciclo de vida. Brazilian Journal of Aquatic Science and Technology. 10(2):1-11.

Perez, J.A.A. 2007. Áreas de exclusão da pesca demersal em áreas profundas da costa brasileira. In: Prates, A. P. \& Blanc, D. (Eds.) Áreas aquáticas protegidas como instrumento da gestão pesqueira. Série Áreas Protegidas do Brasil. Brasília: MMA. 201-216pp.
Perez, J.A.A. \& Pezzuto, P. R. 2006. A pesca de arrasto de talude no sudeste e sul do Brasil: tendências da frota nacional entre 2001 e 2003. Boletim do Instituto de Pesca. 32(2):127-150.

Perez, J. A. A. \& Wahrlich, R. 2005. A bycatch of the gillnet monkfish Lophius gastrophysus fishery off southern Brazil. Fisheries Research. 72:81-95.

Perez, J.A.A.; Pezzuto, P.R.; Wahrlich, R \& Soares, A.L.S. 2009 (no prelo). Deepwater fisheries in Brazil: history, status and perspectives. Latin American Journal of Aquatic Research., 37(2):000-000.

Perez, J. A. A.; Pezzuto, P. R. \& Andrade, H. A. 2005. Biomass assessment of the monkfish Lophius gastrophysus stock exploited by a new deep-water fishery in southern Brazil. Fisheries Research. 72:149-162.

Perez, J. A. A.; Wahrlich, R.; Pezzuto, P.; Schwingel, P.; Lopes, F. R. A. \& Rodrigues-Ribeiro, M. 2003. Deep-sea Fishery off Southern Brazil: Recent Trends of the Brazilian Fishing Industry. Journal of Northwest Atlantic Fishery Science. 31:1-18.

Perez, J.A.A.; Pezzuto, P.R.; Andrade, H.A.; Schwingel, P.R.; Rodrigues-Ribeiro, M. \& Wahrlich, R. 2002. O ordenamento de uma nova pescaria direcionada ao peixe-sapo (Lophius gastrophysus) no sudeste e sul do Brasil. Notas Técnicas da FACIMAR. 6:6583.

Pezzuto, P. \& Dias, M. C. 2007. Estrutura populacional e reprodução dos camarões-de-profundidade (Aristeidae) no talude do Sudeste e Sul do Brasil. Relatório Técnico apresentado à 5a Sessão Ordinária do Subcomitê Científico do Comitê Consultivo Permanente de Gestão dos Recursos Demersais de Profundidade (CPG/Demersais)/SEAP/PR. DOC 21 SCCCPG 05 2007. Itajaí, SC, 11-13/06/2007.

Pezzuto, P.R.; Perez, J.A.A. \& Wahrlich, R. $2006 a$. Deep-sea shrimps (Decapoda: Aristeidae): new targets of the deep-water trawling fishery in Brazil. Brazilian Journal of Oceanography. 54: 123-134.

Pezzuto, P.R.; Perez, J.A.A. \& Wahrlich, R. 2006b. O ordenamento das pescarias de caranguejos-deprofundidade (Chaceon spp.) (Decapoda: Geryonidae) no sul do Brasil. Boletim do Instituto de Pesca. 32(2):229-247.

Pezzuto, P.R.; Perez, J.A.A.; Wahrlich, R.; Vale, W.G. \& Lopes, F.R.A. 2002. Análise da pescaria dos caranguejos-de-profundidade no sul do Brasil-Anos 2001-2002. Ações Prioritárias ao Desenvolvimento da Pesca e Aqüicultura no Sul do Brasil. Convênio Ministério da Agricultura, Pecuária e Abastecimento (MAPA) - Universidade do Vale do Itajaí MAPA/ SARC/DPA/03/2001 e MAPA/SARC/DENACOOP/ $176 / 2002$.

Pires, D. 2007. The azooxanthellate coral fauna of Brazil. In: George, R.Y. \& Cairns, S.D. (Eds.). Conservation 
and adaptive management of seamount and deepsea coral ecosystems. Miami: Rosenstiel Schooll of Marine and Atmospheric Sciences, University of Miami. 265-272pp.

Quinn II, T. J. \& Deriso, R. B. 1999. Quantitative Fish Dynamics. New York: Oxford University Press. 523p.

Rainer, S.F. 1992. Diet of prawns from the continental slope of north-western Australia. Bulletin of Marine Science. 50(2):258-274.

Roberts, C. M. 2002. Deep impact: the rising toll of fishing in the deep sea. Trends in Ecology and Evolution. 17(5):242-245.

Sardà, F.; Company, J.B. \& Maynou, F. 2003. Deepsea shrimp Aristeus antennatus Risso 1816 in the Catalan Sea, a review and perspectives. Journal of Northwest Atlantic Fishery Science. 31:127-136.

Sardà, F.; Calafat, A.; Flexas, M.M.; Tselepides, A.; Canals, M.; Espino, M. \& Tursi, A. 2004. An introduction to Mediterranean deep-sea biology. Scientia Marina. 68(3):7-38.

Serejo, C. S.; Young, P. S.; Cardoso, I. C.; Tavares, C.; Rodrigues, C. \& Almeida, T. C. 2007. Abundância, diversidade e zonação dos crustáceos no talude da costa central do Brasil (11 $\left.1{ }^{\circ}-22^{\circ} \mathrm{S}\right)$ coletados pelo Programa REVIZEE/ Score Central: prospecção pesqueira. In: Costa, P. A. S.; Olavo. G. \& Martins, A. S. (Eds.) Biodiversidade da fauna marinha profunda na costa central brasileira. Rio de Janeiro: Museu Nacional. 133-162pp.

Sparre, P. \& Venema, S.C. 1997. Introdução à avaliação de mananciais de peixes tropicais. Roma: FAO Documento Técnico sobre as Pescas. 404p.

Suman, A.; Wudianto, F. \& Bintoro, G. 2006. Species composition, distribution, and potential yield of deep sea shrimp resources in the Western Sumatera of the Indian Ocean EEZ of Indonesian Waters. Indonesian Fisheries Research Journal. 12(2):2-10.

Tavares, C. R. \& Serejo, C. S. 2007. Taxonomy of Aristeidae (Dendrobranchiata: Penaeoidea) from the central coast of Brazil, collected by the Revizee program, between 19o and 22oS. Zootaxa, 1585:144.

Thompson, H. C.; Arragut, R. N. \& Thompson, M. H. 1977. Relationship of scarlet prawns (Plesiopenaeus edwardsianus) to a benthic oil deposit of the northwest coast of Aruba. Environmental Pollution. 13(4):239-253. 